ISSN 1112-9867

http://www.jfas.info

\title{
APPLICATION OF BLIND DECONVOLUTION AND LUCY-RICHARDSON DECONVOLUTION FOR IMAGE DEBLURRING
}

\author{
M. Z. Abang, M. S. Ramli and S. A. Halim* \\ Center of Mathematical Studies, Univesiti Teknologi MARA, 40450 Shah Alam, Selangor, \\ Malaysia
}

Published online: 17 October 2017

\begin{abstract}
Image is a picture that has been created and stored in electronic form. In digital world, there are various component of effect can interrupt the quality of image and hence produced blurred image. The objectives of this paper are to implement two deblurring algorithms of Blind Deconvolution and Lucy-Richardson Deconvolutionto deblura set of images and evaluate the performance of the two algorithms. There are four main processes involved: data collection; image blurring process, deblurring process and image quality evaluation. The results show that by using Blind Deconvolution, the process of deblurring method give better results compared with Lucy-RichardsonDeconvolution. The deblurring process hashigh impact and very useful in astronomical and medical fieldin order to produce an accurate analysis that based on images such as CT scan and MR images.
\end{abstract}

Keywords: image deblurring; blind deconvolution; Lucy-Richardsondeconvolution; Gaussian blur; MATLAB.

Author Correspondence, e-mail: suhaila@tmsk.uitm.edu.my

doi: http://dx.doi.org/10.4314/jfas.v9i5s.17 


\section{INTRODUCTION}

Nowadays, various applications in medical industry, astronomy, physics, chemistry, forensics, remote sensing, manufacturing and defence often rely upon images to store, display and provide information to us. But, there are many challenges that the scientist and engineers faced in order to extract useful and valuable information from raw image data to be used further for other purposes [1].

In order to retrieve useful information from an image accurately, image processing can be performed. Image processing is a process of converting an image that is normally in digital form and performing some steps and procedures. This process is aims to enhance the quality of an image. The input and the output is the same image with certain characters associated with the image. An image is two dimensional signals where set signal processing method are applied to the image and involves a signal dispensation.

One of the functions in image processing is image deblurring. Image deblurring can be defined as removed distortion from image to retrieve the original image. The purpose of image deblurring is to recover the degraded image that comes in many forms such as motion blur, and noise.The degradation can be morphemic distortions (including turbulence and aerosol scattering), optical aberrations (out of focus blur), sensor blur (resulting from spatial averaging at photosites), motion blur (camera shake or movement of the object in scene) and noise (shot noise and quantization) [2].Moreover, a very good estimation is possible to come up with the actual blurring function. The function can undo the blurring process to restore the original image for motion blur cases. The best solution for the image where it is corrupted by noise is to compensate for the degradation it caused.There are four different ways to restore the image such as Inverse Filter, Weiner Filtering, Wavelet Restoration and Blind Deconvolution.

In [3]mentioned that one of the most important operations in image processing is removal of blur from an image.It considered as pre-processing operation for all applications because image interrogation which is significantly easier if the image is high quality. The removal of blur is called image deconvolution because the point spread function (PSF) can be represents the blur and the PSF is deconvolved from blurred image in order to compute the restored image.Due to inherent diffraction effects, the point gets spread out.The PSF is the response of 
imaging system to a point object or point source. The general term for PSF is a system's impulse response and it being the impulse response of a focused optical system.

In [1] mentioned that degradation consists of two sources that arise during image capturing that are noise and blurring effect. Blurring leads to the spreading of edges which causing loss of sharpness and contrast in the image.The process can be seen as low pass filtering. Blurring in an image can happen either due to the relative motion between the scene and camera or imaging system itself.

Deblurring is the way toward expelling obscuring from pictures such as obscure brought on by defocus abnormality or movement obscure. The obscure is commonly displayed as the convolution of an occasionally space or time-shifting point spread capacity with a theoretical sharp information picture [4]. There is where both the sharp information picture which is to be recuperated and the point spread capacity are obscure. This is a case of a backwards issue. In all cases, there is deficient data in the obscured picture to particularly decide a conceivable unique picture, making it a not well postured issue. Moreover, the obscured picture contains extra clamour which muddles the undertaking of deciding the first picture. This is for the most part illuminated by the utilization of a regularization term to try to take out doubtful arrangements $[5]$.

Image deconvolution process of restoring the image is not aware of the escape.In this situation, the blur kernel is unknown and also the task also requires basic blur kernel estimation. This process is commonly referred to as a blind deconvolution [6]. Deconvolution is a calculation based process used to turn around the impacts of convolution on recorded data.The idea of deconvolution is broadly utilized as a part of the strategies of flag handling and picture preparing. Since these procedures are thus generally utilized as a part of numerous logical and building disciplines, deconvolution finds numerous applications [7].

In mentioned that many blind deconvolution existing research focuses on a single fuzzy seed recovered for the entire image [1]. Deblurring methods that handle different proposals usually rely on multiple frames. Although vague uniform is valid for a limited set of camera motion, it is also far from satisfactory when the scene can contain multiple objects moving independently. The statistic of the blurry image and image estimated is capitalized by using this technique and it is an iterative approach to converge on the correct seeing parameter.The basic task of image 
deblurring is to de-convolute the blurred image with PSF in order to describe the distortion. The first step is, the actual image is degraded by using the Degradation Model and it can be done by Gaussian Filter. Gaussian Filter is low-pass filter that can be used to blur in image. For the edges of degraded image, the ringing effect occurs due to the high frequency drop-off. This can be detected using edge detection methods. By using edge trapping, it should remove the ringing effect before the restoration. After the effect was removed, Blind Deconvolution algorithm is applied to the blurred image.

In utilised Blind Deconvolution algorithm to deblur the degraded images using PSF of various sizes.It shows that the method gives better performance for average blur.

Another method of deblurring the image is by using Lucy-Richardson Algorithm. It can be used effectively when the PSF is known, but little or no information is available for the noise. The blurred and noisy image is restored by the iterative, accelerated, damped Lucy-Richardson algorithm. In [9] mentioned that an adaptive Richardson-Lucy deconvolution algorithm using local extreme filtering. The local extreme filtering is used to extract the edge map of the image which contains definition on smooth regions and then the edge map and blurred image are both used as inputs for deblurring algorithm. The ringing artifact can be reduced for each time of iteration.A shaper deblurred image without ringing can be obtained. The main problem is that some information about the missing details are present in blurred images, but the information is hidden and can only be recovered after the details of the process of blurred images is known.

In this paper, the algorithms for image deblurring are applied on still images.In addition, some other information about the techniques is also explained with brief introduction to the basic problem of image deblurring.

In real problem, ability to extract maximal information from an image is useful. But, it is quite impossible to obtain an image without blur because it is expensive. The missing details in blurred image are due to hidden information, the information needs to be recovered and processed for future purposes. One of the challenges in recovering the information is to make the image deblurring algorithm reliable and efficient to restore the original image. This paper aims to implement two deblurring algorithms of Blind Deconvolution and Lucy-Richardson on test images and hence compare the performances of both methods using image quality metrics of mean absolute error (MAE) and root mean square error (RMSE).

The following sections in this paper are organised as follows. Section 2gives an overview of 
two image debluring methods that are Lucy-Richardson Deconvolution and Blind Deconvolution.Section 3 presents the detail processes in methodology involved in this paper. Section 4 discusses the results and the performances of the deblurring algorithms. Finally Section 5 has drawn the conclusion.

\section{IMAGE DEBLURRING METHOD}

\subsection{Lucy-Richardson Deconvolution}

The Richardson-Lucy deconvolution is an iterative procedure for recovering an image that has been blurred by a known PSF. It was named after William Richardson and Leon Lucywho described it independently. The algorithm maximizes the like hood that the resulting image, when convolved with the PSF, is an instance of the blurred image.

Lucy-Richardson method has builds up an iteration expectation maximization deconvolution method based on a Bayesian framework, $p(X \mid Y)=p(Y \mid X) \frac{p(X)}{p(Y)}$ where $p(X \mid Y)$ is the possibility probability, $p(Y \mid X)$ is the posteriori probability, and $p(X)$ is a prior model image. Given that $p(Y \mid X)=\prod_{v} \frac{\left|H^{*} X(v)^{Y(v)} \cdot e^{-\left(H^{*} X\right)(v)}\right|}{Y(v) !}$ where $X, Y, H: \mathfrak{R}^{3} \rightarrow \mathfrak{R}, v \in \mathfrak{R}^{3}$. The $H$ is the convolution provides a way multiplying together two arrays of numbers.

\subsection{Blind Deconvolution}

Blind deconvolution is a method to recover the sharp version of a blurred image when the blur kernel is unknown [10].In [11]mentioned that Blind Deconvolution can be used wisely and effectively when there is no information or detail about the blurring and noise. The blind deconvolution applies some of the adaptation of the original Lucy-Richardson algorithm.

\section{METHODOLOGY}

In this paper, there involved several processes in research methodology in the process of image deblurring using Blind Deconvolution and Lucy-Richardson algorithms as shown in Fig. 1. 


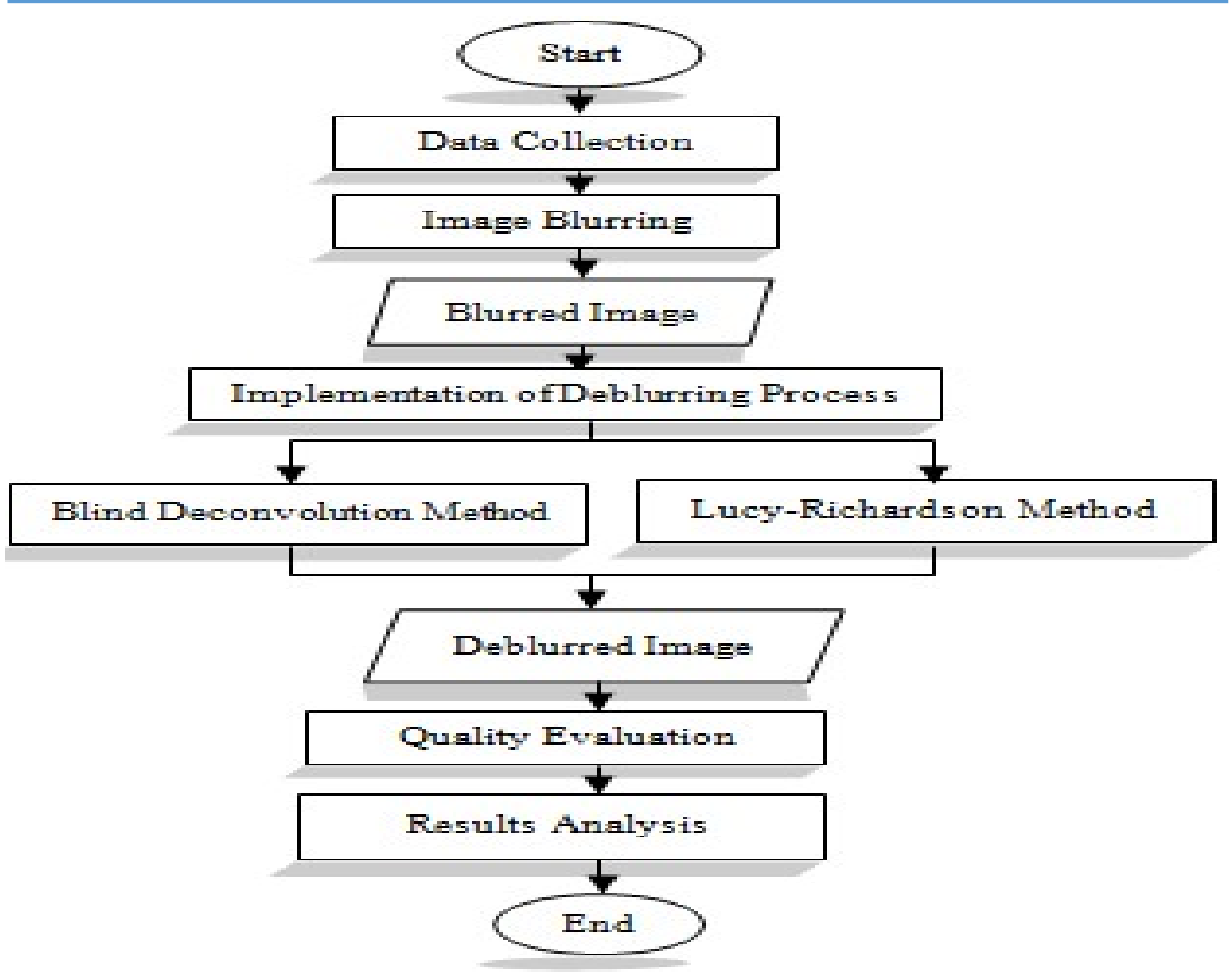

Fig.1.Processes involved in research methodology

For data collection, a set of test images from standard database is extracted from [12]. The dimensions of the test images are $512 \times 512$ pixels and in gray scale as shown in Fig. 2. There are 12 images used in the experimental process. 


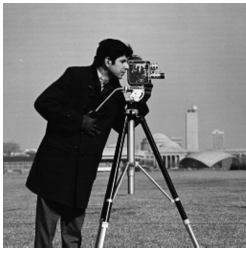

(a) Cameraman

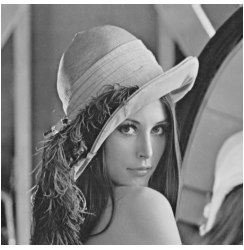

(e) Lena

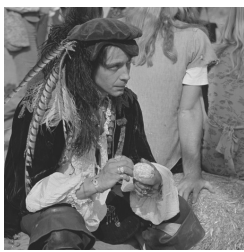

(i) Pirate

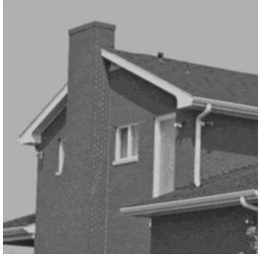

(b) House

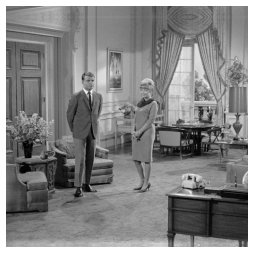

(f) Livingroom

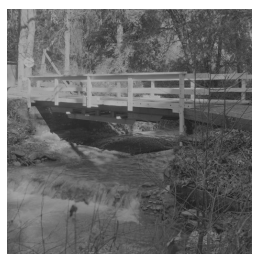

(j) Walkbridge

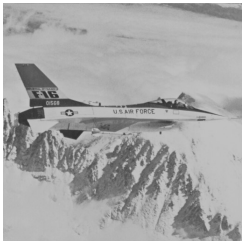

(c) Jetplane

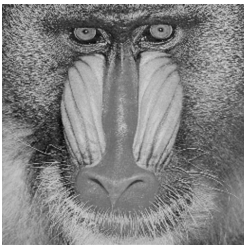

(g) Mandril_gray

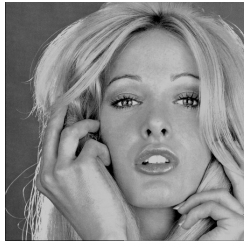

(k) Woman_blonde

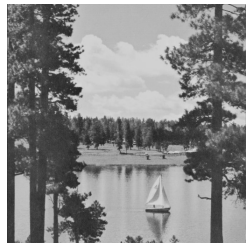

(d) Lake

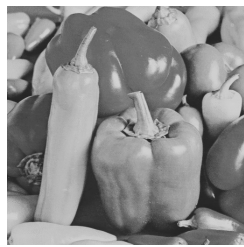

(h) Peppers_gray

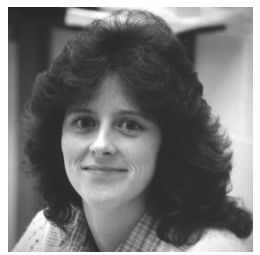

(1) Woman_darkhair

Fig.2.Test images

The next step is image blurring process. All the images are embedded with three different parameters filter sizes of Gaussian blur that purposely embedded for the performance comparison on thedeblurring methods. The three experimented parameters are named Gaussian 1, Gaussian 2 and Gaussian 3. By using the same standard deviation of 10 pixels of the Gaussian blur, the Gaussian 1 represents the blurred image with Gaussian Blur of filter size [7 7]. The Gaussian 2 represents the blurred image with Gaussian Blur of filter size [10 10]. The Gaussian 3 represents the blurred image with Gaussian Blur of filter size [13 13]. As the filter size increases, the blurring effect on the image also increases and produces more blurred image.

Then, two methods of Blind Deconvolution and Lucy-Richardson Deconvolution are implemented in order to deblur the blurred image.

Finally, MAE and RMSE are calculated to measure the performance of the methods. The MAE is used to measure the closeness of the prediction or forecast is to the true values. The MAE is given by, MAE $=\frac{1}{n} \sum_{i=1}^{n}\left|e_{i}\right|$ where $n$ is the number pixel in image [13]. The average of the absolute error $e_{i}=\left|f_{i}-y_{i}\right|$, with the prediction value, $f_{i}$ and the true value of the image is $y_{i}$. 
The RMSE presents a standard metric for model errors and calculated for the data set using $\mathrm{RMSE}=\sqrt{\frac{1}{n} \sum_{i=1}^{n}\left(e_{i}\right)^{2}}$ [13].At the point when both measurements are calculated, the RMSE is by definition never has smaller than the MAE value.

All the processes are implemented using MATLAB R2013a software with the processor Intel® Core ${ }^{\mathrm{TM}}$ i76700HQ CPU @ 2.60GHz of 64-bit Operating System, x64based processor.

\section{RESULTS AND DISCUSSION}

Fig. 3 shows a sample of Cameraman that has been blurred with the three parameters and hence produced blurred image.Additionally, the results of deblurred image from each deblurring process are also shown.

Blur Blurred Image $\quad$ Deblurred Image

Parameter

Gaussian 1

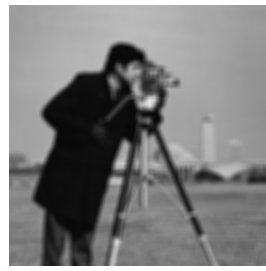

Gaussian 2

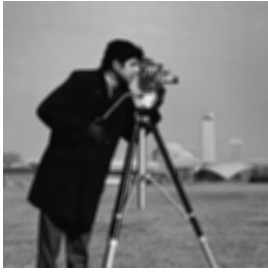

Gaussian 3

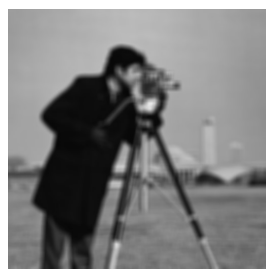

Lucy-Richardson
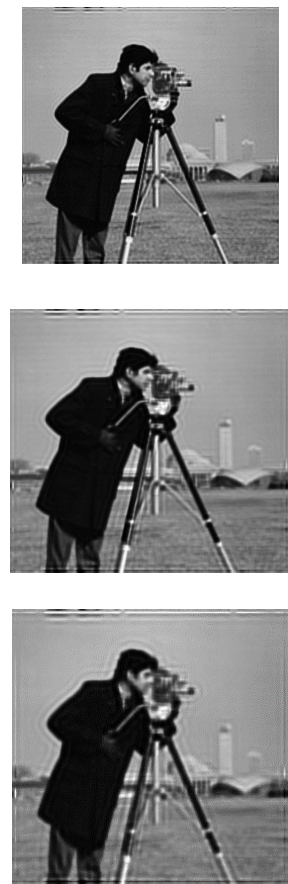

\section{Blind Deconvolution}
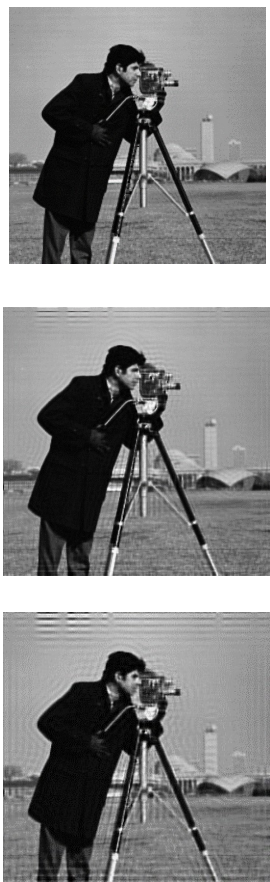

Fig.3.Image quality before and after deblurring process

Table 1, Table 2 and Table 3 show the results of Blind Deconvolution and Lucy-Richardson for all the sample of test images with different Gaussian parameters using MAE and RMSE respectively. 
Table 1.Lucy-Richardson (LR) and Blind Deconvolution (BD) with Gaussian 1

\begin{tabular}{ccccc}
\hline \multirow{2}{*}{ Image Data } & \multicolumn{2}{c}{ MAE } & \multicolumn{2}{c}{ RMSE } \\
& LR & BD & LR & BD \\
\hline cameraman & 2.3738 & 1.7186 & 4.3044 & 3.2652 \\
house & 1.722 & 0.6888 & 3.1107 & 1.4387 \\
jetplane & 1.8468 & 1.4798 & 3.799 & 2.999 \\
lake & 3.4541 & 2.4144 & 5.4074 & 4.3827 \\
lena & 2.8609 & 1.8387 & 4.6809 & 3.5049 \\
livingroom & 4.0697 & 2.7896 & 6.0363 & 4.7624 \\
mandril_gray & 5.1924 & 3.7217 & 6.9101 & 5.8694 \\
peppers_gray & 2.6134 & 1.8252 & 4.3915 & 3.3776 \\
pirate & 3.5073 & 2.4572 & 5.4159 & 4.3752 \\
walkbridge & 3.2247 & 2.3796 & 5.3724 & 4.3386 \\
woman_blonde & 4.1359 & 2.8132 & 5.6522 & 4.4966 \\
woman_darkhair & 2.0117 & 1.0445 & 3.423 & 2.0381 \\
\hline
\end{tabular}

Based on Table 1, it can be observed that BD gives better performance than LR based on lower value of compared with RMSE for blurred image with Gaussian 1. 
Table 2.Lucy-Richardson (LR) and Blind Deconvolution (BD) with Gaussian 2

\begin{tabular}{ccccc}
\hline \multirow{2}{*}{ Image Data } & \multicolumn{2}{c}{ MAE } & \multicolumn{2}{c}{ RMSE } \\
& LR & BD & LR & BD \\
\hline cameraman & 3.4142 & 2.7239 & 5.2041 & 4.6375 \\
house & 2.6377 & 2.2581 & 4.2069 & 3.7768 \\
jetplane & 2.4773 & 2.0986 & 4.5697 & 4.029 \\
lake & 4.4185 & 3.6805 & 6.1305 & 5.6717 \\
lena & 3.7789 & 3.1476 & 5.511 & 5.016 \\
livingroom & 5.0731 & 3.9683 & 6.8115 & 6.0077 \\
mandril_gray & 6.4628 & 5.15 & 7.6064 & 6.9264 \\
peppers_gray & 3.2973 & 2.8097 & 5.1553 & 4.6854 \\
pirate & 4.418 & 3.5618 & 6.1778 & 5.5301 \\
walkbridge & 3.9342 & 3.2372 & 6.1106 & 5.3963 \\
woman_blonde & 4.8652 & 4.4336 & 6.2725 & 5.9497 \\
woman_darkhair & 2.6313 & 2.2283 & 4.1715 & 3.7728 \\
\hline
\end{tabular}

The BD also gives better performance than LR based on Table 2 for blurred image with Gaussian 2. It also gives the same performance results based on Table 3 for Gaussian 3. 
Table 3.Lucy-Richardson (LR) and Blind Deconvolution (BD) with Gaussian 3

\begin{tabular}{ccccc}
\hline \multirow{2}{*}{ Image Data } & \multicolumn{2}{c}{ MAE } & \multicolumn{2}{c}{ RMSE } \\
& LR & BD & LR & BD \\
\hline cameraman & 4.175 & 4.0024 & 5.6795 & 5.7184 \\
house & 3.2441 & 3.9988 & 4.9147 & 5.4811 \\
jetplane & 3.0182 & 2.8636 & 5.1008 & 4.9882 \\
lake & 5.2965 & 4.9852 & 6.639 & 6.562 \\
lena & 4.5822 & 4.7674 & 6.1186 & 6.3756 \\
livingroom & 5.8121 & 5.28 & 7.2787 & 7.035 \\
mandril_gray & 7.2761 & 6.5068 & 7.9735 & 7.7125 \\
peppers_gray & 3.9073 & 4.0169 & 5.7168 & 5.8664 \\
pirate & 5.1769 & 4.8391 & 6.7028 & 6.5281 \\
walkbridge & 4.4585 & 4.0642 & 6.5706 & 6.234 \\
woman_blonde & 5.6268 & 6.0195 & 6.7568 & 6.9498 \\
woman_darkhair & 3.2126 & 3.7652 & 4.7317 & 5.1317 \\
\hline
\end{tabular}

By comparing the results in the three tables, results in Table 3 produce the highest error amongst the Table 1 and Table 2. This is due to the larger filter size been applied during blurring process and cause more blurred image.

When the filter sizes of Gaussian blur become larger, this indicates large convolution mask is implemented. This process could increases the effect of the neighbouring pixels in the image and hence the image quality is reduces as compared to the original value of the image.

\section{CONCLUSION}

In this paper, image deblurring is divided into two methods which are Blind Deconvolution and Lucy-Richardson. In order to evaluate the quality of images, MAE and RMSE are calculated. The results have shown that by using Blind Deconvolution method give better results as compared to using Lucy-Richardson. Thus, the objectives in this paper which are applied and compare the quality of images have been achieved. As for recommendation, it is suggested to implement the deblurring methods with variety of blurring effects in order to 
analyse the performance and robustness of the deblurring methods based on image quality.

\section{ACKNOWLEDGEMENTS}

We would like to thanks to Faculty of Computer and Mathematical Sciences, Universiti Teknologi MARA (UiTM) Shah Alam for giving us opportunity to conduct this study, provide facilities and financial support in supporting this study.

\section{REFERENCES}

[1] Krahmer F, Lin Y Z, MacAdoo B, Ott K, Wang J K, Widemann D, Wohlberg B. Blind image deconvolution: Motion blur estimation. IMA Preprints Series, 2006, 21:33-35

[2] Gunturk B. K., Li. X. Image restoration: Fundamentals and advances. Florida: CRC Press, 2012

[3] Sonka M., Hlavac V., Boyle R. Image processing, analysis, and machine vision. Boston: Cengage Learning, 2014

[4] Jia J. Single image motion deblurring using transparency. In IEEE Conference on Computer Vision and Pattern Recognition, 2007, pp. 1-8

[5] Vincent O, Folorunso O. A descriptive algorithm for sobel image edge detection. In Informing Science and IT Education Conference, 2009, pp. 97-107

[6] Harmeling S, Hirsch M, Sra S, Scholkopf B. Online blind deconvolution for astronomical imaging. In IEEE International Conference on Computational Photography, 2009, pp. 1-7

[7] Krishnan D, Fergus R. Fast image deconvolution using hyper-Laplacian priors. In Advances in Neural Information Processing Systems, 2009, pp. 1033-1041

[8] Saini S, Himral L. Image processing using blind deconvolution deblurring technique. International Journal of Applied Engineering and Technology, 2014, 4(2):115-124

[9] Wu J L, Chang C F, Chen C S. An adaptive Richardson-Lucy algorithm for single image deblurring using local extrema filtering. Journal of Applied Science and Engineering. 2013, 16(3):269-276

[10] Kang K, Cao Y, Wang Z F. A novel approach based on differential evolution for blind deconvolution. In 8th International Conference on Advanced Computational Intelligence. 2016, pp. 112-116

[11] Shakeel M S, Kang W. Efficient blind image deblurring method for palm print image. In IEEE International Conference on Identity, Security and Behavior Analysis, 2015, pp. 1-7 


[12] ImageProcessingPlace.com. Image 2017,

http://www.imageprocessingplace.com/root_files_V3/image_databas es.htm.

[13] Chai T, ad Draxler R R. Root mean square error (RMSE) or mean absolute error (MAE)?-Arguments against avoiding RMSE in the literature. GeoscientificModel Development. 2014, 7(3):1247-1250

How to cite this article:

Abang M Z, Ramli M S, Halim S A. Application of blind deconvolution and lucy-richardson deconvolution for image deblurring. J. Fundam. Appl. Sci., 2017, 9(5S), 232-244. 